\title{
Totally 3D-endoscopic patch closure of aorto-right ventricular fistula
}

Yuichiro Fukumoto, MD, ${ }^{\mathrm{a}}$ Soh Hosoba, MD, PhD, ${ }^{\mathrm{a}, \mathrm{b}}$ Yoshihiro Goto, MD, ${ }^{\mathrm{a}}$ and Toshiaki Ito, MD, PhD, Toyohashi and Nagoya, Japan

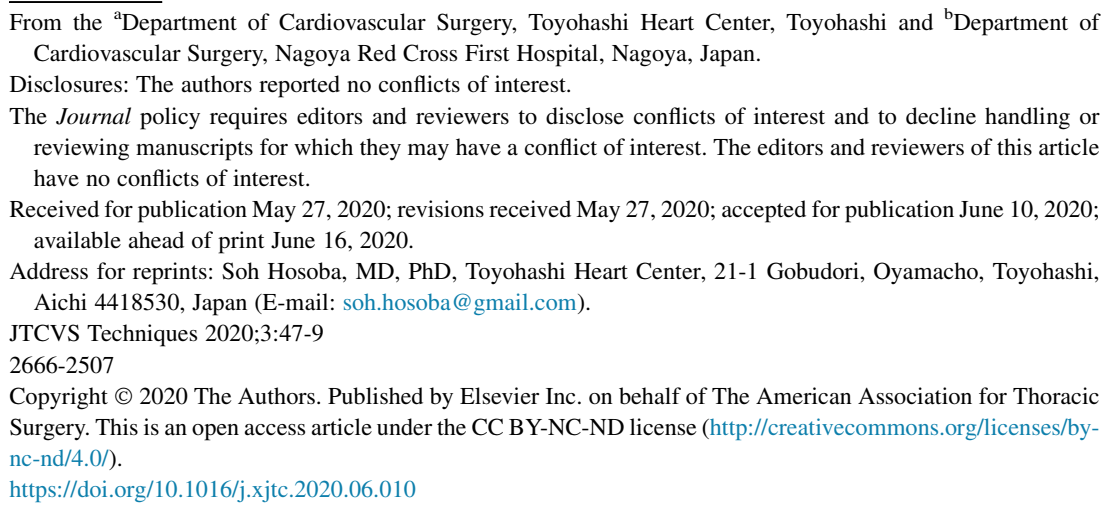

Video clip is available online.

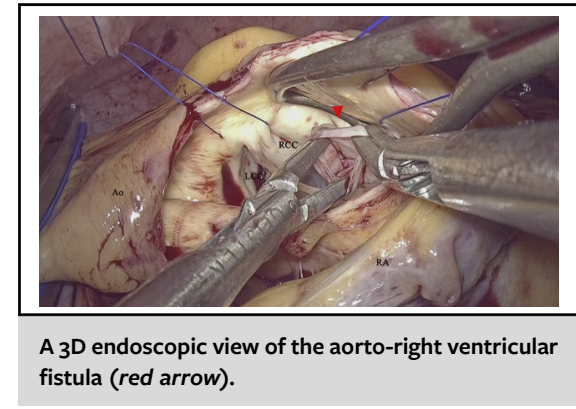

CENTRAL MESSAGE

A right lateral mini-thoracotomy approach using 3D endoscopy is feasible for treating aorto-right ventricular fistula.

See Commentaries on pages 50 and 52.
Aorto-right ventricular fistula (ARVF) is an extremely rare malformation that can lead to heart failure if left untreated. Therefore, surgical repair is recommended, but a suitable approach remains a matter of debate. Here we report a case of totally $3 \mathrm{D}$ endoscopic minimally invasive repair of AVRF.

\section{CLINICAL SUMMARY}

A 26-year-old otherwise healthy male presented with exertional dyspnea and occasional palpitation for 3 months. Physical examination revealed a 4/6 continuous murmur at the right parasternal area in the fourth intercostal space. Transthoracic echocardiography (TTE) showed a left-to-right shunt from the aorta to the right ventricle $(\mathrm{Qp} / \mathrm{Qs}=1.9)$ (Figure 1,A). Computed tomography demonstrated sinus of Valsalva aneurysm (SVA) as a cause of the shunt (Figure 1, $B$ and $C$ ). There was no other congenital cardiac abnormality. Once the patient provided written informed consent, he underwent repair of the ARVF through a right lateral mini-thoracotomy with a totally 3D-endoscopic 3-port minimally invasive approach (Figure 2, A).

The operation was performed under general anesthesia with left lung ventilation. The patient was placed in 30-degree left lateral decubitus position with the right arm up. A 10-mm trocar was inserted for a 3D endoscope through the fourth intercostal space on the mid-axillary line. A main 3-cm incision was made at the fourth intercostal space. A 5-mm trocar for a left-hand port was placed at the second intercostal space. Both ports were on the anterior axillary line. A soft tissue retractor was applied on the main port without rib spreading. Cardiopulmonary bypass was established through the right groin.

After achieving hypothermia and ventricular fibrillation, the ascending aorta was cross-clamped. Cardiac arrest was achieved with selective antegrade cardioplegia delivered through an aortotomy. The ARVF was seen to arise from a right sinus of Valsalva aneurysm (Figure 2, B). The aneurysm was resected at the attachment to the aortic wall, and then an expanded polytetrafluoroethylene patch was sewn over the defect with everting mattress sutures (Video 1). Transesophageal echocardiography demonstrated no shunt flow between the right ventricle and aorta. The aortic cross-clamp time, cardiopulmonary bypass time, and operation time were 90, 132, and 177 minutes, respectively.

The patient did not require transfusion and was extubated in 2 hours. He was transferred to the floor on postoperative day 1 . He had an uneventful recovery and was discharged to home on postoperative day 4 . There was no residual 


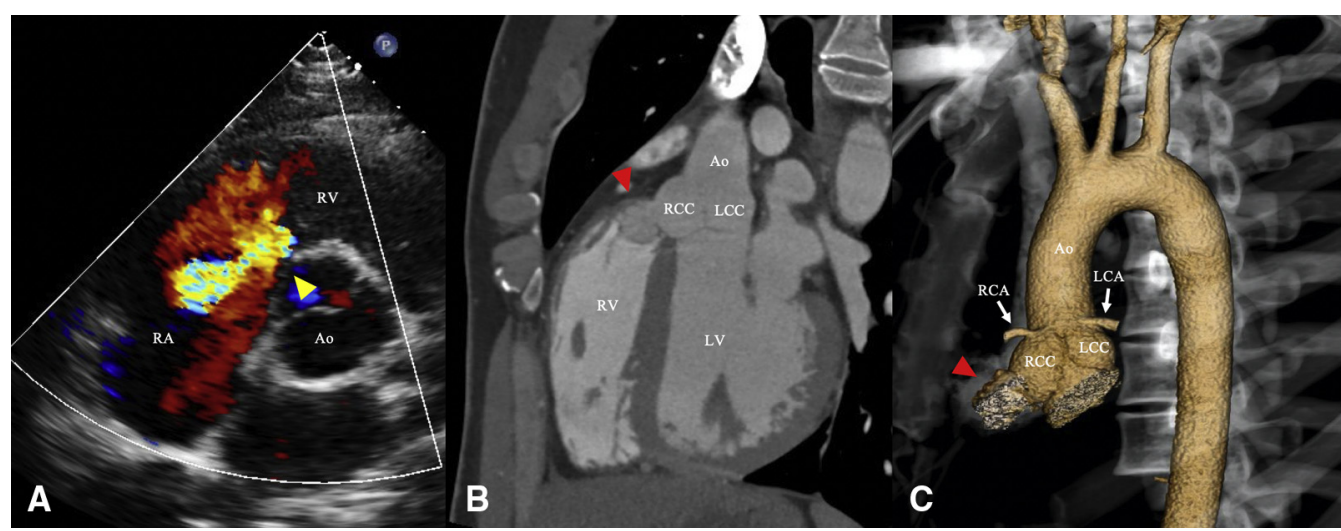

FIGURE 1. (A) Preoperative transthoracic echocardiography showing a continuous shunt flow from the aorta to the right ventricle (yellow arrow). (B and C) Contrast-enhanced computed tomography (B) and volume-rendering image (C) demonstrating a sinus of Valsalva aneurysm from the right coronary sinus (red arrow). $R V$, Right ventricle; $R A$, right atrium; $A o$, aorta; $R C C$, right coronary cusp; $L C C$, left coronary cusp; $L V$, left ventricle; $R C A$, right coronary artery; $L C A$, left coronary artery.

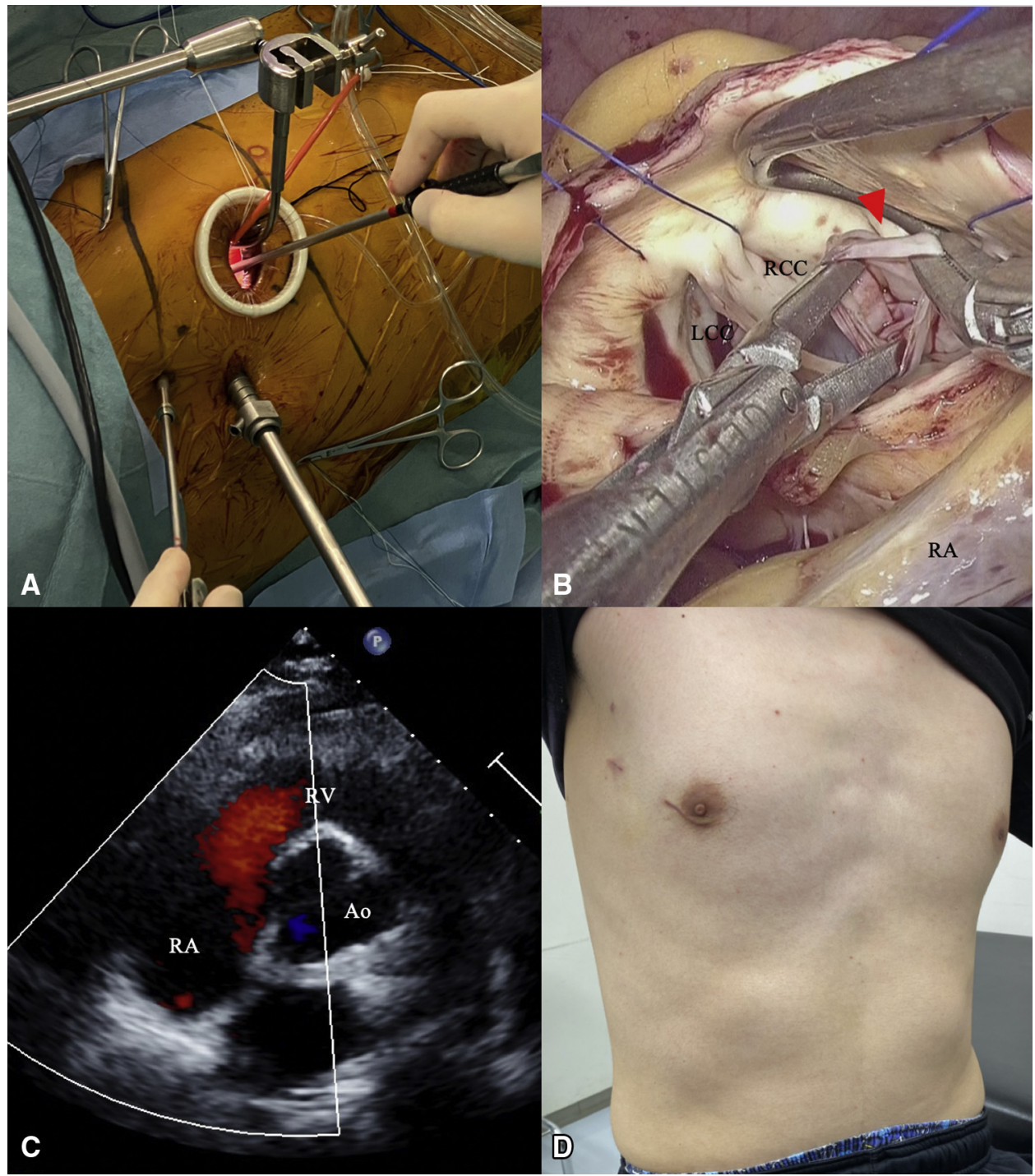

FIGURE 2. (A) The 3-port setup for a right anterolateral mini-thoracotomy approach using a 3D endoscope. The main, second, and camera port incisions were made at the fourth, third, and fourth intercostal spaces, respectively. (B) Aorto-right ventricular fistula (red arrow) arising from the right sinus of Valsalva through the 3D endoscope. (C) There was no residual shunt on the postoperative echocardiogram. (D) A well-healed main incision and 2 trocar wounds were observed at the outpatient visit. $R C C$, Right coronary cusp; $L C C$, left coronary cusp; $R A$, right atrium; $R V$, right ventricle; $A o$, aorta. 


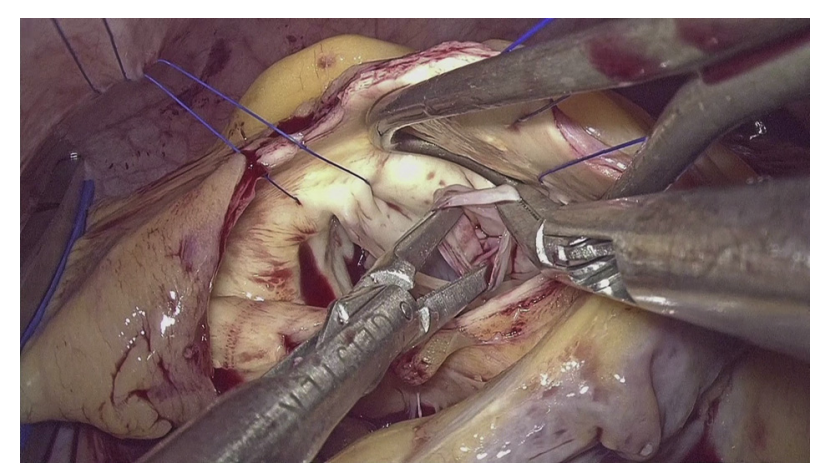

VIDEO 1. Video demonstrating an intraoperative view of the ARVF and the repair technique. Video available at: https://www.jtcvs.org/article/S26 66-2507(20)30288-1/fulltext.

shunt or aortic insufficiency on TTE, and the small incision was well healed at the 3-month postoperative visit (Figure 2, $C$ and $D$ ).

\section{DISCUSSION}

Aortoventricular fistula is an extremely rare malformation characterized by the aberrant connection between the aorta and the right or left ventricle. The causes of aortoventricular fistula include aortic dissection, chest trauma, infective endocarditis, and aortic valve surgery, with rupture of a congenital or acquired SVA the most common cause. ${ }^{1}$ Most originate in the right coronary sinus and are associated with the right ventricle, as was the case for our patient. Although a good long-term outcome of surgical repair of ruptured SVA has been reported, aortic regurgitation might occur after this surgical repair. Especially in young patients, a minimally invasive approach via a lateral thoracotomy may be beneficial in allowing easier access if redo surgery is needed in the future.

According to Ruttkay and colleagues ${ }^{2}$ reported that 3D-endoscopic view provides better visualization of deep structures compared with conventional sternotomy. Reproducibility and safety of the totally 3D-endoscopic mitral valve surgery and nonvalvular surgery via a right anterolateral mini-thoracotomy approach also have been reported, ${ }^{3,4}$ with a recent report demonstrating the efficacy of totally 3D-endoscopic AVRs. ${ }^{5}$ We have performed more than 1000 totally endoscopic cases, including 800 mitral valve surgeries and 200 aortic valve surgeries. We also have performed aorto-right atrial or ventricular fistula repair via a median sternotomy. We evaluated imaging data considering endocarditis or trauma as a possible etiology, and determined that the endoscopic view will provide ample exposure of the orifice of the fistula. Our approach in this case was similar to the report of totally 3D-endoscopic AVR. These suggest that 3D-endoscopic approach is useful for nonvalvular periaortic surgery. In our case, we achieved favorable exposure of the fistula site by retracting the aortic wall, allowing us to sufficiently resect the aneurysmal area and facilitating the patch repair of the defect. The minimally invasive approach may also offer shorter intensive care unit and hospital stays. ${ }^{4,5}$ Our cross-clamp time might be slightly longer than expected with a conventional approach, but the operation time remained under 3 hours, and the patient made an uneventful recovery.

To the best of our knowledge, this is the first reported case of totally 3D-endoscopic repair of ARVF. The patient had an excellent result with this approach. Such a minimally invasive approach may be technically feasible to treat ARVF.

\section{References}

1. Mve Mvondo C, Pugliese M, Dailor EM. Surgical repair of a giant aorto-left ventricular fistula. Tex Heart Inst J. 2019;46:133-5.

2. Ruttkay T, Götte J, Walle U, Doll N. Minimally invasive cardiac surgery using a 3D high-definition endoscopic system. Innovations. 2015;10:431-4.

3. Ito T, Maekawa A, Hoshino S, Hayashi Y, Sawaki S, Yanagisawa J, et al. Threeport (one incision plus two-port) endoscopic mitral valve surgery without robotic assistance. Eur J Cardiothorac Surg. 2017;51:913-8.

4. Deng L, Zhang GW, Liu ZH, Meng WX, Liu HY. Totally thoracoscopic surgery for atrial myxomas resection and atrial septal defect repair. Eur Rev Med Pharmacol Sci. 2017;21:569-75.

5. Tokoro M, Sawaki S, Ozeki T, Orii M, Usui A, Ito T. Totally endoscopic aortic valve replacement via an anterolateral approach using a standard prosthesis. Interact Cardiovasc Thorac Surg. 2020;30:424-30. 